\title{
Compressibility Corrections to Closure Approximations for Turbulent Flow Simulations
}

\author{
L. D. Cloutman
}

February 1, 2002

U.S. Department of Energy

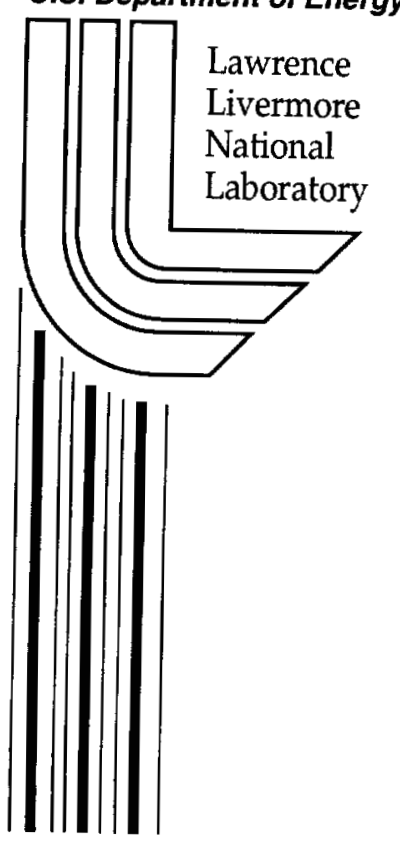

Approved for public release; further dissemination unlimited 


\section{DISCLAIMER}

This document was prepared as an account of work sponsored by an agency of the United States Government. Neither the United States Government nor the University of California nor any of their employees, makes any warranty, express or implied, or assumes any legal liability or responsibility for the accuracy, completeness, or usefulness of any information, apparatus, product, or process disclosed, or represents that its use would not infringe privately owned rights. Reference herein to any specific commercial product, process, or service by trade name, trademark, manufacturer, or otherwise, does not necessarily constitute or imply its endorsement, recommendation, or favoring by the United States Government or the University of California. The views and opinions of authors expressed herein do not necessarily state or reflect those of the United States Government or the University of California, and shall not be used for advertising or product endorsement purposes.

This work was performed under the auspices of the U.S. Department of Energy by the University of California, Lawrence Livermore National Laboratory under Contract No. W-7405-Eng-48.

This report has been reproduced directly from the best available copy.

Available electronically at http://www.doc.gov/bridge

Available for a processing fee to U.S. Department of Energy

And its contractors in paper from

U.S. Department of Energy

Office of Scientific and Technical Information P.O. Box 62

Oak Ridge, TN 37831-0062

Telephone: (865) 576-8401

Facsimile: (865) 576-5728

E-mail: reports@adonis.osti.gov

Available for the sale to the public from

U.S. Department of Commerce

National Technical Information Service

5285 Port Royal Road

Springfield, VA 22161

Telephone: (800) 553-6847

Facsimile: (703) 605-6900

E-mail: orders@ntis.fedworld.gov

Online ordering: http://www.ntis.gov/ordering.htm

OR

Lawrence Livermore National Laboratory

Technical Information Department's Digital Library

http://www.llnl.gov/tid/Library.html 
Lawrence Livermore National Laboratory Report

UCRL-ID-??????

February 2002

\title{
COMPRESSIBILITY CORRECTIONS TO CLOSURE APPROXIMATIONS FOR TURBULENT FLOW SIMULATIONS
}

\author{
Lawrence D. Cloutman
}

\begin{abstract}
We summarize some modifications to the usual closure approximations for statistical models of turbulence that are necessary for use with compressible fluids at all Mach numbers. We concentrate here on the gradient-flux approximation for the turbulent heat flux, on the buoyancy production of turbulence kinetic energy, and on a modifcation of the Smagorinsky model to include buoyancy. In all cases, there are pressure gradient terms that do not appear in the incompressible models and are usually omitted in compressible-flow models. Omission of these terms allows unphysical rates of entropy change.
\end{abstract}




\section{Introduction}

A major source of uncertainty in most turbulence models is the use of the gradient-flux (or gradient-transport) approximation to calculate the turbulent fluxes of chemical species and heat. That is,

$$
\overline{f^{\prime} \mathbf{u}^{\prime}}=-\frac{\mu_{t}}{\tilde{\rho} \mathrm{Sc}_{f}} \nabla \bar{f},
$$

where $\tilde{\rho}=\langle\rho\rangle$ is the averaged density, $\mu_{t}$ is the eddy viscosity, and $\mathrm{Sc}_{f}$ is the turbulent Schmidt or Prandtl number for the quantity $f$. Angled brackets denote the averaging operator. We take $f$ to be one of the scalar functions that describe fluid flow, such as internal energy $I$, temperature $T$, or a species mass fraction $Y_{\alpha}$. Here a prime indicates a turbulent fluctuation defined by $f^{\prime}=f-\bar{f}$, and a bar indicates a mass-weighted average: $\bar{f}=\langle\rho f\rangle / \tilde{\rho}$. At this level of approximation, the Reynolds stress usually is modeled by a slightly more complex gradient-flux approximation,

$$
\tilde{\rho} \overline{\mathbf{u}^{\prime} \mathbf{u}^{\prime}}=-\mu_{t}\left[\nabla \overline{\mathbf{u}}+(\nabla \overline{\mathbf{u}})^{\mathrm{T}}-\frac{2}{3} \mathrm{U} \nabla \cdot \overline{\mathbf{u}}\right]+\frac{2}{3} \tilde{\rho} \mathcal{K} \mathrm{U} \equiv-\mathrm{T}_{\mathrm{t}}+\frac{2}{3} \tilde{\rho} \mathcal{K} \mathrm{U},
$$

where $\mathcal{K}$ is the turbulence kinetic energy density, $\mathbf{u}$ is the velocity, and $U$ is the unit tensor. This form has the important properties that its trace is twice the turbulence kinetic energy density, and it preserves the tensor invariance of the mean flow momentum equation. However, it is well-known that the gradient-flux approximation is often seriously in error and even can have the wrong sign (for example, $[1,2,3,4]$ ). So far, satisfactory closure approximations for this counter-gradient phenomenon have not been reported. A negative eddy viscosity was an early proposal [5]. However, this proposal would make the mathematical initial-boundary problem for the mean flow ill-posed, so a different approach must be used.

A more meaningful approach is to introduce terms in addition to the gradient-diffusion term. One of the main goals of this report is to propose just such a counter-gradient term for the turbulent heat flux, which also has implications for the creation of $\mathcal{K}$ by buoyancy. By counter-gradient, we don't necessarily mean the net flux has the opposite sign from the regular gradient term, just that there are one or more terms that oppose it. In such a case, the gradient term alone does not accurately represent the net turbulent flux.

In spite of considerable effort by Deardorff $[3,4]$ and unpublished work by myself, attempts to derive an improved closure model from the governing equations have not provided satisfactory results. For now, we shall abandon this approach and instead use a kinematic model of turbulent mixing to derive the most basic of the counter-gradient terms. The studies based on the governing equations suggest the presence of additional terms, but those are beyond the scope of this report.

The original governing equations are presented in Section 2. The averaged equations and turbulent flux transport equations are presented in Section 3. Section 4 presents the 
kinematic model that shows the existence and form of a very fundamental counter-gradient term. Section 5 discusses the generalized adiabatic exponents and how to evaluate them. Section 6 discusses the creation of turbulence kinetic energy by buoyancy. Section 7 provides a modification to the Smagorinsky model to account for buoyancy. Section 8 contains the conclusions.

\section{Governing Equations}

We assume that the details of turbulent flows can be described to a sufficiently high degree of accuracy by the multicomponent Navier-Stokes equations. We assume the fluid is a mixture of species described by the single velocity (mass weighted) representation.

Mass conservation is expressed by the continuity equation for each species $\alpha$ :

$$
\frac{\partial \rho_{\alpha}}{\partial t}+\nabla \cdot\left(\rho_{\alpha} \mathbf{u}\right)=-\nabla \cdot \mathbf{J}_{\alpha}+R_{\alpha},
$$

where $\rho_{\alpha}$ is the density of species $\alpha, t$ is time, and $R_{\alpha}$ is the rate at which species $\alpha$ is created by chemical reactions. The "exact" diffusional mass fluxes must be computed from a complicated theory (for example, $[6,7,8,9,10,11]$ ) but it will suffice for our present purpose to consider Fick's law,

$$
\mathbf{J}_{\alpha}=-\rho D \nabla\left(\rho_{\alpha} / \rho\right)=-\rho D \nabla Y_{\alpha}
$$

where $\rho$ is the total density, $Y_{\alpha}=\rho_{\alpha} / \rho$ is the mass fraction, and $D$ is the diffusivity. We assume $D$ is independent of species. Equation 3 may be summed over species to obtain the total continuity equation

$$
\frac{\partial \rho}{\partial t}+\nabla \cdot(\rho \mathbf{u})=0
$$

By definition, the diffusional and reactive terms are constrained to sum to zero.

The momentum equation is

$$
\frac{\partial(\rho \mathbf{u})}{\partial t}+\nabla \cdot(\rho \mathbf{u u})=\sum_{\alpha} \rho_{\alpha} \mathbf{F}_{\alpha}-\nabla P+\nabla \cdot \mathrm{T}=\rho \mathrm{g}-\nabla P+\nabla \cdot \mathrm{T},
$$

where $\mathbf{F}_{\alpha}$ is the external body force on species $\alpha$, which we will take to be the gravitational acceleration $\mathbf{g}, P$ is the pressure, and $\mathrm{T}$ is the stress tensor

$$
\begin{gathered}
\mathrm{T}=\mu\left[\nabla \mathbf{u}+(\nabla \mathbf{u})^{T}\right]+\mu_{1}(\nabla \cdot \mathbf{u}) \mathrm{U} \\
=\mu\left[\nabla \mathbf{u}+(\nabla \mathbf{u})^{T}-\frac{2}{3}(\nabla \cdot \mathbf{u}) \mathrm{U}\right]+\mu_{\mathrm{b}}(\nabla \cdot \mathbf{u}) \mathrm{U} .
\end{gathered}
$$

Here $U$ is the unit tensor, $\mu$ is the coefficient of viscosity, $\mu_{1}$ is the second coefficient of viscosity, and $\mu_{\mathrm{b}}$ is the bulk viscosity. We normally assume $\mu_{1}=-2 \mu / 3$ (that is, zero bulk viscosity). 
For reasons discussed elsewhere [12], we choose to express energy conservation in terms of the specific thermal internal energy $I$ :

$$
\frac{\partial(\rho I)}{\partial t}+\nabla \cdot(\rho I \mathbf{u})=-P \nabla \cdot \mathbf{u}+\mathrm{T}: \nabla \mathbf{u}-\nabla \cdot \mathbf{q}+\sum_{\alpha} H_{\alpha} R_{\alpha}+\sum_{\alpha} \mathbf{F}_{\alpha} \cdot \mathbf{J}_{\alpha}
$$

where $\mathrm{q}$ is the diffusional heat flux, and $H_{\alpha}$ is the heat of formation of species $\alpha$. The heat flux is another complicated function, and we choose to consider just the sum of Fourier's law and enthalpy diffusion:

$$
\mathbf{q}=-K \nabla T+\sum_{\alpha} h_{\alpha} \mathbf{J}_{\alpha}
$$

where $K$ is the thermal conductivity, and $h_{\alpha}$ is the enthalpy of species $\alpha$. We emphasize that whatever model, turbulent or laminar, is used to evaluate the species fluxes, the same fluxes must be used in both the species diffusion and the enthalpy diffusion term. Failure to do so will result in a violation of the second law of thermodynamics.

The thermal equation of state is assumed to be the sum of partial pressures for a mixture of ideal gases:

$$
P=\sum_{\alpha} R \rho_{\alpha} T / M_{\alpha}
$$

where $R$ is the universal gas constant, and $M_{\alpha}$ is the molecular weight of species $\alpha$. For present purposes, the caloric equation of state is assumed to be

$$
\rho I=\sum_{\alpha} \rho_{\alpha} I_{\alpha}(T)=\sum_{\alpha} \frac{R \rho_{\alpha} T}{M_{\alpha}\left(\gamma_{\alpha}-1\right)}
$$

where $I_{\alpha}$ is the ionic species specific thermal internal energy. However, for combustion applications, the $I_{\alpha}(T)$ must include rotational, vibrational, and excited states. An example is provided by the JANAF tables $[13,14,15]$. Other applications may require still other constitutive relations that are beyond the scope of the present study.

\section{The General Reynolds Averaged Equations}

The first step in constructing a turbulence model is to select an averaging operator appropriate to the problem at hand. The choice of averaging operator defines the mean flow as some particular function of the total flow. The turbulent component is the difference between the total flow and the mean flow. We use the ensemble average in this study.

We denote the averaging operator by angular brackets or by a tilde above certain variables, for example

$$
\langle\rho(\mathbf{r}, t)\rangle \equiv \tilde{\rho}(\mathbf{r}, t)
$$


The turbulent component $\rho^{\prime}(\mathbf{r}, t) \equiv \rho-\tilde{\rho}$ has a zero average value. If $f$ is any variable except $\rho$ or $P$, the average is mass-weighted, denoted by an overbar:

$$
\langle\rho(\mathbf{r}, t) f(\mathbf{r}, t)\rangle=\tilde{\rho}(\mathbf{r}, t) \bar{f}(\mathbf{r}, t) .
$$

Let us begin by writing down the equations for the mean flow. Averaging equations 3 , 5,6 , and 8 , we obtain [12]

$$
\begin{gathered}
\frac{\partial \tilde{\rho} \overline{Y_{\alpha}}}{\partial t}+\nabla \cdot\left[\tilde{\rho} \overline{Y_{\alpha}} \overline{\mathbf{u}}+\tilde{\rho} \overline{\mathbf{u}^{\prime} Y_{\alpha}^{\prime}}+\left\langle\mathbf{J}_{\alpha}\right\rangle\right]=\left\langle R_{\alpha}\right\rangle \\
\frac{\partial \tilde{\rho}}{\partial t}+\nabla \cdot(\tilde{\rho} \overline{\mathbf{u}})=0 \\
\frac{\partial(\tilde{\rho} \overline{\mathbf{u}})}{\partial t}+\nabla \cdot\left[\tilde{\rho} \overline{\mathbf{u}} \overline{\mathbf{u}}+\tilde{\rho} \overline{\mathbf{u}^{\prime} \mathbf{u}^{\prime}}-\langle\mathrm{T}\rangle\right]=\sum_{\alpha}\left\langle\rho_{\alpha} \mathbf{F}_{\alpha}\right\rangle-\nabla \tilde{P}=\tilde{\rho} \overline{\mathbf{g}}-\nabla \tilde{P}
\end{gathered}
$$

and

$$
\begin{aligned}
& \frac{\partial(\tilde{\rho} \bar{I})}{\partial t}+\nabla \cdot\left[\tilde{\rho} \bar{I} \overline{\mathbf{u}}+\tilde{\rho} \overline{I^{\prime} \mathbf{u}^{\prime}}-\langle K \nabla T\rangle+\sum_{\alpha}\left\langle h_{\alpha} \mathbf{J}_{\alpha}\right\rangle\right] \\
= & \sum_{\alpha} H_{\alpha}\left\langle R_{\alpha}\right\rangle-\langle P \nabla \cdot \mathbf{u}\rangle+\langle\mathbf{T}: \nabla \mathbf{u}\rangle+\sum_{\alpha}\left\langle\mathbf{F}_{\alpha} \cdot \mathbf{J}_{\alpha}\right\rangle .
\end{aligned}
$$

The last term in equation 17 vanishes in the special case of $\mathbf{F}_{\alpha}=\mathbf{g}$. We shall assume gravity is the only body force in the remainder of this report. Note that up to this point the equations are exact. However, we now have a serious closure problem because there are several unknown turbulence correlations that appear in these equations.

In this report, we shall be concerned primarily with $\overline{\mathbf{u}^{\prime} \mathbf{u}^{\prime}}, \overline{I^{\prime} \mathbf{u}^{\prime}}$, and $\overline{Y_{\alpha}^{\prime} \mathbf{u}^{\prime}}$. The transport equations for the two covariances and the turbulence kinetic energy (half the trace of $\left.\overline{\mathbf{u}^{\prime} \mathbf{u}^{\prime}}\right)$ are

$$
\begin{aligned}
& \frac{\partial \tilde{\rho} \overline{Y_{\alpha}^{\prime} \mathbf{u}^{\prime}}}{\partial t}+\nabla \cdot\left[\tilde{\rho} \overline{\mathbf{u}} \overline{Y_{\alpha}^{\prime} \mathbf{u}^{\prime}}+\tilde{\rho} \overline{Y_{\alpha}^{\prime} \mathbf{u}^{\prime} \mathbf{u}^{\prime}}\right]+\tilde{\rho} \overline{\mathbf{u}^{\prime} \mathbf{u}^{\prime}} \cdot \nabla \overline{Y_{\alpha}}+\tilde{\rho} \overline{Y_{\alpha}^{\prime} \mathbf{u}^{\prime}} \cdot \nabla \overline{\mathbf{u}}=\left\langle\rho Y_{\alpha} \mathbf{g}\right\rangle-\tilde{\rho} \bar{Y} \bar{\alpha} \overline{\mathbf{g}} \\
&-\left\langle Y_{\alpha} \nabla P\right\rangle+\overline{Y_{\alpha}} \nabla \tilde{P}+\left\langle\mathbf{u} R_{\alpha}\right\rangle-\overline{\mathbf{u}}\left\langle R_{\alpha}\right\rangle-\left\langle\mathbf{u} \nabla \cdot \mathbf{J}_{\alpha}\right\rangle+\overline{\mathbf{u}} \nabla \cdot\left\langle\mathbf{J}_{\alpha}\right\rangle+\left\langle Y_{\alpha} \nabla \cdot \mathrm{T}\right\rangle-\overline{Y_{\alpha}} \nabla \cdot\langle\mathrm{T}\rangle,(18) \\
& \quad \frac{\partial \tilde{\rho} \overline{I^{\prime} \mathbf{u}^{\prime}}}{\partial t}+\nabla \cdot \tilde{\rho} \overline{\mathbf{u} I^{\prime} \mathbf{u}^{\prime}}+\nabla \cdot \tilde{\rho} \overline{I^{\prime} \mathbf{u}^{\prime} \mathbf{u}^{\prime}}+\tilde{\rho} \overline{I^{\prime} \mathbf{u}^{\prime}} \cdot \nabla \overline{\mathbf{u}}+\tilde{\rho} \overline{\mathbf{u}^{\prime} \mathbf{u}^{\prime}} \cdot \nabla \bar{I} \\
&=\langle\rho I \mathbf{g}\rangle- \tilde{\rho} \bar{I} \overline{\mathbf{g}}-\langle I \nabla P\rangle+\bar{I} \nabla \tilde{P}-\langle\mathbf{u} P \nabla \cdot \mathbf{u}\rangle+\overline{\mathbf{u}}\langle P \nabla \cdot \mathbf{u}\rangle+\langle I \nabla \cdot \mathrm{T}\rangle-\bar{I} \nabla \cdot\langle\mathrm{T}\rangle \\
&+\langle\mathbf{u} \nabla \cdot K \nabla T\rangle-\overline{\mathbf{u}} \nabla \cdot\langle K \nabla T\rangle+\langle\mathbf{u}(\mathbf{T}: \nabla \mathbf{u})\rangle-\overline{\mathbf{u}}\langle\mathrm{T}: \nabla \mathbf{u}\rangle \\
&+\sum_{\alpha}\left[H_{\alpha}\left(\left\langle R_{\alpha} \mathbf{u}\right\rangle-\left\langle R_{\alpha}\right\rangle \overline{\mathbf{u}}\right)-\left\langle\mathbf{u} \nabla \cdot\left(h_{\alpha} \mathbf{J}_{\alpha}\right)\right\rangle+\overline{\mathbf{u}} \nabla \cdot\left\langle h_{\alpha} \mathbf{J}_{\alpha}\right\rangle\right],
\end{aligned}
$$

and

$$
\begin{gathered}
\frac{\partial \tilde{\rho} \mathcal{K}}{\partial t}+\nabla \cdot\left[\tilde{\rho} \mathcal{K} \overline{\mathbf{u}}+0.5 \tilde{\rho} \overline{\left(\mathbf{u}^{\prime} \cdot \mathbf{u}^{\prime}\right) \mathbf{u}^{\prime}}\right]+\tilde{\rho} \overline{\mathbf{u}^{\prime} \mathbf{u}^{\prime}}: \nabla \overline{\mathbf{u}} \\
=\langle\rho \mathbf{g} \cdot \mathbf{u}\rangle-\tilde{\rho} \overline{\mathbf{g}} \cdot \overline{\mathbf{u}}-[\langle\mathbf{u} \cdot \nabla P\rangle-\overline{\mathbf{u}} \cdot \nabla \tilde{P}]+[\langle\mathbf{u} \cdot(\nabla \cdot \mathrm{T})\rangle-\overline{\mathbf{u}} \cdot(\nabla \cdot\langle\mathrm{T}\rangle)] .
\end{gathered}
$$




\section{A Kinematic Closure Model}

In principle, the most accurate way to calculate the second order correlations such as $\overline{Y_{\alpha}^{\prime} \mathbf{u}^{\prime}}$ should be to solve the transport equation 18. However, the problem of closure again requires that several new correlations have to be modeled. Furthermore, the number of component equations for the $\overline{Y_{\alpha}^{\prime} u_{i}^{\prime}}$ is the number of species times the number of dimensions in the calculations. For example, a three-dimensional calculation with 12 species would require carrying 36 transport equations and associated memory arrays just for the species-velocity correlations. Therefore, there is a potentially huge savings in computer memory requirements and computational effort in using an algebraic closure.

Neither Deardorff nor I have had a clear success in using the covariance transport equations to derive an algebraic closure model for the heat and mass fluxes. The biggest impediment is the need to introduce other ad hoc closure assumptions at some point. However, simple kinematic models include pressure gradient terms in a natural way. We discuss three such models. The first two are merely suggestive for our present purposes, but are nonetheless instructive and provide support for the final closure approximations. The third model provides a more detailed and complete "derivation" of the closure for the case of a multicomponent fluid in an arbitrary flow.

\subsection{Stellar Convection Zones}

The simplest example of a gradient-flux model with a counter-gradient term is found in the most elementary theory of convection that has been used widely in models of stellar atmospheres and interiors for several decades. Stellar models are usually one-dimensional, and the mean flow equations are found by averaging the Navier-Stokes equations in spherical coordinates over surfaces of constant radius. Convective instability occurs in a chemically homogeneous stratified fluid whenever the superadiabatic excess

$$
\Delta \nabla T \equiv\left[\frac{\partial \bar{T}}{\partial r}-\left(\frac{\partial \bar{T}}{\partial r}\right)_{\mathrm{ad}}\right]<0 .
$$

This is the well-known Schwarzschild criterion. The subscript "ad" denotes the adiabatic gradient. Kaniel and Kovetz [16] show that the Schwarzschild criterion for convective instability in a star may be written in terms of the sign of the entropy gradient, and we note that $\Delta \nabla \bar{T}$ is proportional to the entropy gradient for a chemically homogeneous ideal gas.

In the usual one-dimensional stellar interior and atmosphere models, only the radial component of the turbulent heat flux $\overline{I^{\prime} \mathbf{u}^{\prime}}$ is nonzero, and it is usually estimated from the standard mixing length theory (SMLT) $[17,18]$. The SMLT long has been the standard model of turbulent convection in astrophysics, and its shortcomings are well known. Its 
foundation is not a dynamical calculation based on the equations of fluid motion, but rather on a "cartoon derivation" in which "turbulent blobs" are assumed to be created, move due to buoyancy forces, and decay within a hydrostatic environment. The SMLT is not really a theory but is more accurately described as a kinematic model of convection. The only dynamics in the SMLT is the estimation of the buoyancy forces on the assumed "blobs."

The heat flux in the SMLT may be written as

$$
\overline{I^{\prime} u_{\tau}^{\prime}}=-A_{\mathrm{SMLT}}\langle v\rangle_{\mathrm{SMLT}} H_{P} C_{P}\left[\frac{\partial \bar{T}}{\partial r}-\left(\frac{\partial \bar{T}}{\partial r}\right)_{\mathrm{ad}}\right] \equiv-A_{\mathrm{SMLT}}\langle v\rangle_{\mathrm{SMLT}} H_{P} C_{P} \Delta \nabla \bar{T},
$$

where $A_{\mathrm{SMLT}}$ is an empirically determined constant, $\langle v\rangle_{\mathrm{SMLT}}$ is a turbulent velocity, $H_{P}$ is the pressure scale height, and $C_{P}$ is the specific heat at constant pressure. The first four factors are equivalent to a kinematic eddy viscosity divided by a turbulent Prandtl number. Since the convective velocity $\langle v\rangle_{\text {SMLT }}$ is proportional to $|\Delta \nabla \bar{T}|^{1 / 2}$, the flux is often written in a form that explicitly shows a $\Delta \nabla \bar{T}^{3 / 2}$ dependence. The importance of equation 22 , however, is that the turbulent heat flux is proportional to the superadiabatic excess gradient $\Delta \nabla \bar{T}$ rather than $\nabla \bar{T}$. It is easy to see why this is so: if the temperature gradient is the adiabatic gradient, adiabatically moving a blob of fluid up or down in pressure equilibrium will not produce a temperature difference between the blob and the ambient medium, hence there is no net convective transfer of heat by an ensemble of upward- and downward-moving blobs. If the adiabatic gradient is less steep (less negative since $r$ increases outward) than the actual gradient, an adiabatically rising blob cools more slowly than its surroundings as it rises, thereby transporting an amount of thermal energy proportional to the difference between the blob temperature and the ambient temperature at the level where the blob merges with the ambient medium. Note that since $\Delta \nabla \bar{T}$ is proportional to the entropy gradient, neglect of the adiabatic term in the turbulent heat flux closure, even for low Mach number "incompressible" flows, can lead to serious errors, including the incorrect sign for the heat and entropy fluxes. That is, a simulation can violate the second law of thermodynamics if the adiabatic term is not included.

The conventional wisdom in other areas of fluid dynamics is that counter-gradient effects are mostly due to pressure gradients. This is consistent with the case of stars in hydrostatic equilibrium discussed above, since we can write

$$
\left(\frac{\partial \bar{T}}{\partial r}\right)_{\mathrm{ad}}=\left(\frac{\Gamma_{2}-1}{\Gamma_{2}}\right) \frac{\bar{T}}{\tilde{P}} \frac{\partial \tilde{P}}{\partial r}
$$

where $\Gamma_{2}$ is the second adiabatic exponent [19]. It reduces to $\gamma$ for gases in the absence of radiation pressure. We propose generalizing equation 22 to

$$
\overline{I^{\prime} \mathbf{u}^{\prime}}=-\frac{\mu_{\mathrm{t}}}{\tilde{\rho} \operatorname{Pr}_{\mathrm{t}}}\left(\nabla \bar{I}-C_{v} \frac{\Gamma_{2}-1}{\Gamma_{2}} \frac{\bar{T}}{\tilde{P}} \nabla \tilde{P}\right)
$$


for multidimensional simulations, where $C_{v}$ is the specific heat at constant volume.

The situation becomes more complex in chemically inhomogeneous systems. Ledoux [20] notes that it is density, not temperature, that is the fundamental determining factor for buoyancy forces, and therefore for the stability of the fluid. The more general condition for convective instability is then

$$
\left(\frac{T}{\rho}\right)\left[\frac{\partial \rho}{\partial r}-\left(\frac{\partial \rho}{\partial r}\right)_{\mathrm{ad}}\right]=\left[\frac{\partial T}{\partial r}-\left(\frac{\partial T}{\partial r}\right)_{\mathrm{ad}}\right]-\frac{T}{M} \frac{d M}{d r}<0,
$$

where $M$ is the mean molecular weight. We shall incorporate this modification into the final model in section 4.3 .

\subsection{A Meteorological Model}

Deardorff $[3,4]$ has studied the counter-gradient flux problem in the context of meteorological flows. Instead of using internal energy, he expresses energy conservation in terms of the potential temperature $\theta_{T}$ defined by

$$
\theta_{T}=T\left(\frac{P_{0}}{P}\right)^{(\gamma-1) / \gamma}
$$

where $P_{0}$ is the pressure of an arbitrary reference state, often the initial condition of the fluid element under consideration. We assume an ideal gas with constant $\gamma$ and $C_{v}$. In that case, $\theta_{T}$ is constant for a Lagrangian fluid element if the flow is purely adiabatic, and the energy equation reduces to $d \theta_{T} / d t=0$. Even for nonadiabatic flows, this choice of variables formally removes the $P d V$ work term from the energy equation, which can be seen by considering equation 8 rewritten in Lagrangian form:

$$
\rho \frac{d I}{d t}=-P \nabla \cdot \mathbf{u}+\mathcal{S}_{I}
$$

where $\mathcal{S}_{I}$ is shorthand for all of the source and diffusion terms. Then the potential temperature equation is

$$
\rho \frac{d \theta_{T}}{d t}=\frac{\theta_{T}}{I} \mathcal{S}_{I}
$$

The averaged potential temperature equation is

$$
\frac{\partial \tilde{\rho} \bar{\theta}_{T}}{\partial t}+\nabla \cdot\left(\tilde{\rho} \bar{\theta}_{T} \overline{\mathbf{u}}\right)=-\nabla \cdot\left[\tilde{\rho} \overline{\theta_{T}^{\prime} \mathbf{u}^{\prime}}\right]+\left\langle\frac{\theta_{T}}{I} \mathcal{S}_{I}\right\rangle \approx \nabla \cdot\left[\frac{\mu_{t}}{\operatorname{Pr}_{\theta_{T}}}\left(\nabla \bar{\theta}_{T}-\gamma_{c} \hat{z}\right)\right]+\left\langle\frac{\theta_{T}}{I} \mathcal{S}_{I}\right\rangle
$$

where $\gamma_{c}$ is an empirical constant and $\hat{z}$ is the unit vector in the vertical direction. The gradient-flux term with $\gamma_{c}=0$ assumes the validity of equation 1 for $f=\theta_{T}$. Since $\nabla \theta_{T}=$ $\left(\theta_{T} \Delta \nabla T\right) / T$, the pressure gradient term in equation 24 is already included in the ordinary gradient-flux approximation for $\overline{\theta_{T}^{\prime} \mathbf{u}^{\prime}}$ with $\gamma_{c}=0$. Deardorff [2] has presented a similar 
derivation that, strictly speaking, is limited to planar atmospheres in hydrostatic equilibrium with low-Mach-number flows.

This result is a strong argument that equation 24 provides a zeroth order approximation to the counter-gradient term, at least for gases for which the polytropic law equation 26 describes adiabatic changes. However, Deardorff points out that atmospheric measurements show the need to include the additional counter-gradient term in the turbulent heat flux in equation 29 with $\gamma_{c} \approx 6.5 \times 10^{-6} \mathrm{~K} / \mathrm{cm}$. He published two attempts to theoretically explain this term by looking at the turbulence transport equations. The first attempt looked at the transport equation for the potential temperature variance [3]. He showed that a combination of the triple correlation and decay terms can produce this additional counter-gradient effect. Deardorff also did a later analysis [4] using the transport equation for the turbulent potential temperature flux and found a rather different result. These arguments, however, are not compelling and further research is needed.

\subsection{A Kinematic Model of Mixing}

Let us consider a simple kinematic model of turbulent mixing much in the spirit of that usually used to derive the Schwarzschild and Ledoux criteria. The pressure-gradient term will arise naturally. Also, it will be clear that the counter-gradient term is appropriate for all flows, not just stratified atmospheres. Figure 1 shows two Lagrangian control volumes of fluid, labelled 1 and 2 . We assume that fluid elements of equal mass, indicated by the circles, exchange positions. The exchange is assumed to be adiabatic and reversible (isentropic), without species diffusion, and with the fluid elements remaining intact until after the exchange. Then the elements are allowed to "dissolve" and mix with the rest of the control volume. The pressure in each control volume is unchanged by this process, but the volume is allowed to change. We now consider the changes made to the contents of the control volumes due to this exchange process. This model is appropriate for use with Schumann's averaging procedure [21] since the control volumes may be identified with computational zones.

We shall assume the following for the equations of state in a multicomponent fluid mixture. First, there are no chemical changes. Second, the total pressure is the sum of the partial pressures of the species. Third, the specific internal energy of the mixture is given by the mass-weighted average of the species internal energies. Unless otherwise specified, the partial pressures may or may not be for an ideal gas, and the species internal energies may be nonlinear functions of temperature and density that are thermodynamically consistent with the thermal equation of state. 


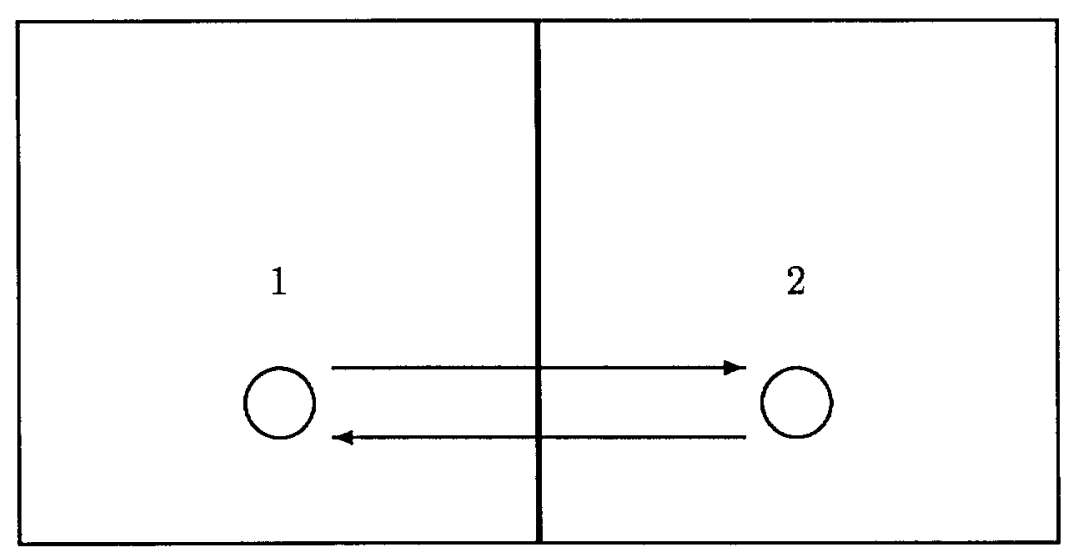

Figure 1. Control volumes for the kinematic model.

We assume the interchange of blobs of volume $d V_{i}$ of equal mass

$$
d m=\rho_{1} d V_{1}=\rho_{2} d V_{2}
$$

Then for each species $\alpha$ we have a mass balance in control volume 1

$$
\breve{V}_{1} \check{\rho}_{\alpha 1}=V_{1} \rho_{\alpha 1}-\rho_{\alpha 1} d V_{1}+\rho_{\alpha 2} d V_{2}=V_{1} \rho_{\alpha 1}+d m\left(Y_{\alpha 2}-Y_{\alpha 1}\right),
$$

where $\check{\rho}_{\alpha 1}$ is the species density after the exchange. Since the total mass of volume 1 is $M_{1} \equiv \breve{V}_{1} \check{\rho}_{1}=V_{1} \rho_{1}$, the change in mass fraction of species $\alpha$ is

$$
\check{Y}_{\alpha 1}=Y_{\alpha 1}+\frac{d m}{M_{1}}\left(Y_{\alpha 2}-Y_{\alpha 1}\right)
$$

This result is consistent with the usual Fick's law closure for the turbulent mass fluxes,

$$
\left\langle\mathrm{J}_{\alpha}\right\rangle=\tilde{\rho} \overline{\mathbf{u}^{\prime} Y_{\alpha}^{\prime}}=-\frac{\mu_{\mathrm{t}}}{\mathrm{Sc}_{\mathrm{t}}} \nabla \bar{Y}_{\alpha}
$$

where $\mathrm{Sc}_{\mathrm{t}}$ is the turbulent Schmidt number. This relation is independent of the equations of state and of the thermodynamic process used in the exchange.

Next consider a stability test assuming the blobs remain intact immediately after the exchange. This implicitly assumes that the pressure equilibration occurs rapidly compared to chemical mixing or heat transfer. We use an asterisk to denote the conditions within the blob after pressure equilibration at the new location, but before it mixes with its new surroundings. Then for the mixture that moved from volume 2 to volume 1 ,

$$
\frac{P_{2}-P_{1}}{P_{1}}=\Gamma_{1} \frac{\rho_{2}-\rho_{2}^{*}}{\rho_{2}},
$$


where, following Chandrasekhar [19], we introduce the generalized adiabatic exponent $\Gamma_{1} \equiv$ $(\partial \ln P / \partial \ln \rho)_{\text {ad }}$, which can be different for each mixture. ${ }^{1}$ We assume that the fractional changes in pressure and density are much less than unity. Similarly, we have for each fluid species

$$
\frac{P_{\alpha 2}^{*}-P_{\alpha 2}}{P_{\alpha 2}}=\Gamma_{1, \alpha} \frac{\rho_{\alpha 2}^{*}-\rho_{\alpha 2}}{\rho_{\alpha 2}} .
$$

We do not assume that the $\Gamma_{1, \alpha}$ are equal. For an ideal gas, $\Gamma_{1}=\gamma$, the usual ratio of specific heats. The next section discusses how to evaluate this parameter for any equation of state.

We assume the total pressure is equal to the sum of partial pressures, for example,

$$
P_{2}=\sum_{\alpha} P_{\alpha 2}
$$

As long as the blob remains intact, all species change their density by the same factor, so $\rho_{\alpha 2}^{*} / \rho_{\alpha 2}=\rho_{2}^{*} / \rho_{2}$ and $Y_{\alpha 2}^{*}=Y_{\alpha 2}$. In general, the pressure fractions will change inside the blob during the adiabatic expansion or compression due to the exchange. However, the constraint

$$
P_{1}=\sum_{\alpha} P_{\alpha 1}=\sum_{\alpha} P_{\alpha 2}^{*}
$$

will be satisfied. Noting that all species in the blob undergo the same fractional density change, solving equation 35 for $P_{\alpha 2}^{*}$, and substituting into equation 37 , we obtain the fractional density change for the blob $d V_{2}$ :

$$
\frac{\rho_{2}^{*}-\rho_{2}}{\rho_{2}}=\frac{P_{1}-P_{2}}{\sum_{\alpha} \Gamma_{1, \alpha} P_{\alpha 2}} .
$$

This equations tells us the new blob density $\rho_{2}^{*}$.

Stability can be determined by comparing the new blob density to the density of its surroundings and the direction of the local pressure gradient. If $\left(P_{2}-P_{1}\right)\left(\rho_{1}-\rho_{2}^{*}\right)>0$, the blob gets further accelerated by buoyancy forces and the fluid is unstable. Otherwise, there is a restoring force on the blob. We note that $\left(P_{2}-P_{1}\right)\left(\rho_{1}-\rho_{2}^{*}\right) \propto-\nabla P \cdot\left[\nabla \rho-(\nabla \rho)_{\text {ad }}\right]$, so we recover the Ledoux criterion, equation 25. A common error in the literature is to test stability by considering the sign of $\nabla P \cdot \nabla \rho$, thereby omitting the adiabatic gradient term. However, it is clear that this simplified form is incomplete and therefore incorrect when applied to compressible fluids regardless of the Mach number.

Now consider the heat flux due to element exchange. The internal energy in volume 1 is given by

$$
\rho_{1} I\left(Y_{\alpha 1}, T_{1}\right)=\sum_{\alpha} \rho_{\alpha 1} I_{\alpha}\left(T_{1}\right)=\rho_{1} \sum_{\alpha} Y_{\alpha 1} I_{\alpha}\left(T_{1}\right)
$$

\footnotetext{
${ }^{1}$ The generalized adiabatic exponents are not constant in general but are weak functions of the thermodynamic state. For our purposes, we can evaluate $\Gamma_{1}$ from the initial state of each fluid element, which is the same level of approximation as the denominators in equation 34 .
} 
Upon an exchange of blobs, the mixture energy balance is

$$
\check{V}_{1} \check{\rho}_{1} I\left(\check{Y}_{\alpha 1}, \check{T}_{1}\right)=V_{1} \rho_{1} I\left(Y_{\alpha 1}, T_{1}\right)-d m \sum_{\alpha}\left[Y_{\alpha 1} I_{\alpha}\left(T_{1}\right)-Y_{\alpha 2} I_{\alpha}\left(T_{2}^{*}\right)\right]
$$

Here we have assumed that the blob from $d V_{2}$ has all species at the same final temperature $T_{2}^{*}$ at the end of the exchange, but before its dissolution. If we note that blob mass fractions are unchanged by the exchange and divide by $M_{1}$, this becomes

$$
\begin{gathered}
I\left(\check{Y}_{\alpha 1}, \check{T}_{1}\right)=I\left(Y_{\alpha 1}, T_{1}\right)-\frac{d m}{M_{1}} \sum_{\alpha}\left[Y_{\alpha 1} I_{\alpha}\left(T_{1}\right)-Y_{\alpha 2} I_{\alpha}\left(T_{2}^{*}\right)\right] \\
=I\left(Y_{\alpha 1}, T_{1}\right)-\frac{d m}{M_{1}}\left[I\left(Y_{\alpha 1}, T_{1}\right)-I\left(Y_{\alpha 2}, T_{2}^{*}\right)\right] .
\end{gathered}
$$

The new internal energy $I\left(\check{T}_{1}\right)$ can be inverted to find the new temperature $\check{T}_{1}$ once we evaluate the $I_{\alpha}\left(T_{2}^{*}\right)$.

We compute $T_{2}^{*}$ from the generalized adiabatic exponent $\Gamma_{2}$ defined by $\Gamma_{2} /\left(\Gamma_{2}-1\right) \equiv$ $(\partial \ln P / \partial \ln T)_{a d}$. Assuming we have $\Gamma_{2}$ for the mixture in control volume 2, we allow $d V_{2}$ to undergo an adiabatic change from $P_{2}$ to $P_{1}$ :

$$
\frac{P_{1}-P_{2}}{P_{2}}=\frac{\Gamma_{2}}{\Gamma_{2}-1} \frac{T_{2}^{*}-T_{2}}{T_{2}}=\frac{\Gamma_{2}}{\Gamma_{2}-1} \frac{I\left(Y_{\alpha 2}, T_{2}^{*}\right)-I\left(Y_{\alpha 2}, T_{2}\right)}{C_{\mathrm{v}, 2} T_{2}}
$$

where $C_{\mathrm{v}, 2} \equiv(\partial I / \partial T)_{\mathrm{v}}$ is the specific heat at constant volume. Note that the second equality is valid, strictly speaking, only for $d I=C_{v} d T$, which neglects $(\partial I / \partial \rho)_{T} d \rho$ for non-ideal caloric equations of state. Solving equation 42 for $I\left(Y_{\alpha 2}, T_{2}^{*}\right)$ and substituting into equation 41 ,

$$
I\left(\check{Y}_{\alpha 1}, \check{T}_{1}\right)=I\left(Y_{\alpha 1}, T_{1}\right)-\frac{d m}{M_{1}}\left[I\left(Y_{\alpha 1}, T_{1}\right)-I\left(Y_{\alpha 2}, T_{2}\right)\right]+\frac{d m}{M_{1}}\left(\frac{\Gamma_{2}-1}{\Gamma_{2}}\right) \frac{C_{\mathrm{v}, 2} T_{2}}{P_{2}}\left(P_{1}-P_{2}\right) .
$$

Noting that the internal energy and pressure differences are proportional to the respective gradients, we obtain our new closure approximation,

$$
\tilde{\rho} \overline{\mathbf{u}^{\prime} I^{\prime}}=-\frac{\mu_{\mathrm{t}}}{P r_{\mathrm{t}}} \nabla \bar{I}+\frac{\mu_{\mathrm{t}} C_{v}}{P r_{\mathrm{t}}} \frac{\Gamma_{2}-1}{\Gamma_{2}} \frac{\bar{T}}{\tilde{P}} \nabla \tilde{P},
$$

which may be compared to equation 24. We have now removed the assumptions of ideal gases and chemical homogeneity.

\section{The Generalized Adiabatic Exponents}

Chandrasekhar [19] (pages $56 \mathrm{ff}$ ) discusses adiabatic processes for general equations of state by introducing the generalized adiabatic exponents $\Gamma_{1}, \Gamma_{2}$, and $\Gamma_{3}$ defined by

$$
\left(\frac{\partial \ln P}{\partial \ln \rho}\right)_{\mathrm{ad}}=\Gamma_{1}
$$




$$
\begin{aligned}
& \left(\frac{\partial \ln P}{\partial \ln T}\right)_{\mathrm{ad}}=\frac{\Gamma_{2}}{\Gamma_{2}-1} . \\
& \left(\frac{\partial \ln T}{\partial \ln \rho}\right)_{\mathrm{ad}}=\Gamma_{3}-1,
\end{aligned}
$$

For the purposes of this report, we shall assume that the adiabatic processes are also isentropic. It is easy to show that the adiabatic exponents are constrained by

$$
\frac{\Gamma_{3}-1}{\Gamma_{1}}=\frac{\Gamma_{2}-1}{\Gamma_{2}}
$$

The new turbulence model requires both $\Gamma_{1}$ and $\Gamma_{2}$. For ideal gases, these are simply the usual ratio of specific heats, $\gamma$. For radiation in the gray one-temperature approximation, $\Gamma_{i}=4 / 3$. For other equations of state, the evaluation is somewhat more complex. Sometimes these exponents are provided as part of equation of state tables, such as with the OPAL equation of state rogers:01, but in most cases the user has to provide them. We shall now show how to evaluate these quantities within a computational fluid dynamics program with any thermodynamically consistent equation of state.

We shall find it convenient to introduce the usual specific volume, $v=1 / \rho$ and to follow the nomenclature and analysis of Sears [23]. Let us assume that we have been given the thermal and caloric equations of state $P(v, T)$ and $I(v, T)$. We begin by defining the isothermal coefficient of expansion

$$
\beta=\frac{1}{v}\left(\frac{\partial v}{\partial T}\right)_{P} \quad\left(=\frac{1}{T} \quad \text { Ideal gas }\right)
$$

and the isothermal compressibility

$$
\kappa=-\frac{1}{v}\left(\frac{\partial v}{\partial P}\right)_{T} \quad\left(=\frac{1}{P} \quad \text { Ideal gas }\right) .
$$

We shall also need to make use of the thermodynamic consistency condition

$$
\left(\frac{\partial I}{\partial v}\right)_{T}+P=T\left(\frac{\partial P}{\partial T}\right)_{v}=\frac{T \beta}{\kappa}
$$

which is derived on page 148 of Sears [23] and on page 36 of Chandrasekhar [19]. It must be emphasized that any realistic pair of equations of state $P(v, T)$ and $I(v, T)$ must obey this consistency condition in order to obtain physically meaningful results.

Now consider the combined first and second laws of thermodynamics for an isentropic (reversible adiabatic) process,

$$
T d s=0=d I_{s}+P d v_{s}=C_{v} d T_{s}+\left[\left(\frac{\partial I}{\partial v}\right)_{T}+P\right] d v_{s},
$$


where $s$ is the specific entropy. The subscript $s$ on the differentials indicates that they are constrained to lie on an isentrope in the $(v, T)$ plane. Collecting terms, we have

$$
-C_{v} \frac{d T_{s}}{d v_{s}}=\rho^{2} C_{v}\left(\frac{\partial T}{\partial \rho}\right)_{s}=\rho T C_{v}\left(\Gamma_{3}-1\right)=\left(\frac{\partial I}{\partial v}\right)_{T}+P=T\left(\frac{\partial P}{\partial T}\right)_{v}=\frac{T \beta}{\kappa} .
$$

The most convenient of those equalities for evaluation of $\Gamma_{3}$ will depend on which thermodynamic derivatives are most readily available in the program.

In order to evaluate $\Gamma_{2}$, we shall reconsider the previous analysis but with $P$ and $T$ taken as the independent variables, so the equations of state are $v(P, T)$ and $I(P, T)$. The combined first and second laws of thermodynamics for an isentropic process becomes

$$
0=d I_{s}+P d v_{s}=\left(\frac{\partial I}{\partial T}\right)_{P} d T_{s}+\left(\frac{\partial I}{\partial P}\right)_{T} d P_{s}+P\left[\left(\frac{\partial v}{\partial T}\right)_{P} d T_{s}+\left(\frac{\partial v}{\partial P}\right)_{T} d P_{s}\right] .
$$

Rearranging terms, we obtain

$$
\frac{T}{P}\left(\frac{\partial P}{\partial T}\right)_{s}=\frac{\Gamma_{2}}{\Gamma_{2}-1}=-\left(\frac{T}{P}\right) \frac{\left(\frac{\partial I}{\partial T}\right)_{P}+P\left(\frac{\partial v}{\partial T}\right)_{P}}{\left(\frac{\partial I}{\partial P}\right)_{T}+P\left(\frac{\partial v}{\partial P}\right)_{T}}=\left(\frac{T}{P}\right) \frac{C_{P}}{P v \kappa-\left(\frac{\partial I}{\partial P}\right)_{T}} .
$$

This reduces correctly to $\gamma /(\gamma-1)$ for an ideal gas. We note that the thermodynamic derivative $(\partial I / \partial P)_{T}$ may be converted into other thermodynamic derivatives using various relationships that are easily derived or found in standard thermodynamics texts. The third adiabatic exponent $\Gamma_{1}$ may be evaluated from the other two using equation 48 .

An alternative formula for computing $\Gamma_{1}$ is trivially obtained by noting that the square of the isentropic sound speed is given by

$$
C^{2} \equiv\left(\frac{\partial P}{\partial \rho}\right)_{s}=\frac{\Gamma_{1} P}{\rho}=\left(\frac{\partial P}{\partial \rho}\right)_{T}+\frac{T\left(\frac{\partial P}{\partial T}\right)_{\rho}^{2}}{\rho^{2}\left(\frac{\partial E}{\partial T}\right)_{\rho}}
$$

Again, this form may be more useful than equations 53 or 55 , depending on which thermodynamic quantities are most readily available.

\section{The Buoyancy Creation Term}

The buoyancy creation term in the turbulence kinetic energy transport equation 20 has been ignored in most published turbulence models because the emphasis has been on shear flows. This term has not been as thoroughly studied as some other aspects of turbulence modeling, and there are some open questions about how to model it. To the best of my knowledge, the earliest published example of an algebraic model for the buoyancy creation term is in 
the one-equation model of Rivard, Butler, and Farmer [24]. This model uses a non-massweighted ensemble average of the compressible multicomponent Navier-Stokes equations, and the form of the buoyancy source term follows directly from the governing equations by using the gradient-flux approximation to the density-velocity correlation. Cloutman [25] extended the model to properly account for the Ledoux criterion for turbulent convection in a study of overshooting from the convective cores of massive stars. This extension was done on an essentially ad hoc basis using a nonmathematical physical argument.

The stability result from section 4.3 suggests that the closure approximation for the buoyancy creation rate of turbulence kinetic energy $\mathcal{K}$ for compressible fluids should be [25, 26]

$\frac{\partial \rho \mathcal{K}}{\partial t}=\mu_{t} \mathcal{B}+\ldots=-C_{B} \frac{\mu_{t}}{\tilde{\rho}^{2}}\left[\nabla \tilde{\rho}-(\nabla \tilde{\rho})_{s}\right] \cdot \nabla \tilde{P}+\ldots=-C_{B} \frac{\mu_{t}}{\tilde{\rho}^{2}}\left[\nabla \tilde{\rho}-\frac{\tilde{\rho}}{\Gamma_{1} \tilde{P}} \nabla \tilde{P}\right] \cdot \nabla \tilde{P}+\ldots$

where $C_{B}$ is a constant that we take to be 1.4 .

In most formulations of closure for the buoyancy source term, the adiabatic gradient term is omitted. This is an egregious error since it affects the local rate of entropy production, up to and including getting the sign wrong. The adiabatic term tends to make the production rate somewhat smaller in magnitude in buoyantly unstable regions while spreading the driving force over a larger region of space.

As this source term is written, it drives turbulence when the fluid is unstable according to the Ledoux criterion (which also includes the Rayleigh-Taylor unstable case as an extreme limit) and damps turbulence in a stable case. It may be argued that this term should not provide damping in stable cases, but should be set to zero [25]. The conventional wisdom is that when this term predicts damping of the turbulence, what happens physically is that the buoyant blobs undergo oscillatory motion rather than monotonically accelerated motion. The existing kinetic energy is no longer affected by buoyancy forces and is dissipated only by viscosity. However, in the Richtmyer-Meshkov instability, turbulence grows regardless of the sign of this term, suggesting that under some conditions the absolute value of it should be used. Clearly, the present closure models may need to be supplemented to account for shock waves or other impulsive accelerations, and taking the absolute value seems to be inappropriate for most problems. Preliminary indications from a very limited set of calculations are that the best comparison with experiments is obtained when we "rectify" $\mathcal{B}$. That is, we calculate $\mathcal{B}$ from equation 57 , but set it to zero if the value is negative. However, this conclusion is tentative, and this question requires additional validation studies for its resolution. Turbulent plumes are a good possibility as the buoyancy effects are important and there is a large body of experimental data available. 


\section{A Modified Smagorinsky Model}

The transport equation 20 for the turbulence kinetic energy can be solved only after suitable closure approximations have been made. This has been done $[26,27]$ using the usual filter function approach to large eddy simulation and the Schumann subgrid-scale approach [21]. The Smagorinsky model for the eddy viscosity $[28,29]$ may be recovered from the modeled $\mathcal{K}$ equation in the limit of steady homogeneous turbulence in which compression effects (that is, the divergence of the mean flow velocity) and buoyancy effects are negligible. The surviving terms have shear creation in balance with viscous dissipation. Under these conditions the turbulence transport equation simplifies to

$$
\frac{D_{\mathrm{t}} \tilde{\rho} \mathcal{K}^{3 / 2}}{L}=\mu_{\mathrm{t}}\left[\nabla \overline{\mathbf{u}}+(\nabla \overline{\mathbf{u}})^{\mathrm{T}}\right]: \nabla \overline{\mathbf{u}} \equiv \mu_{\mathrm{t}} S^{2} .
$$

The term on the left is the viscous decay rate of $\mathcal{K}, D_{\mathrm{t}}$ is a model constant of order unity, and $L$ is the turbulence length scale. We can use the additional closure assumption

$$
\mu_{\mathrm{t}}=A_{\mathrm{t}} \tilde{\rho} L \mathcal{K}^{1 / 2}
$$

to eliminate $\mathcal{K}$, with the result that

$$
\mu_{\mathrm{t}}=\left(\frac{A_{\mathrm{t}}^{3}}{D_{\mathrm{t}}}\right)^{1 / 2} \tilde{\rho} L^{2}\left\{\nabla \overline{\mathbf{u}}:\left[\nabla \overline{\mathbf{u}}+(\nabla \overline{\mathbf{u}})^{\mathrm{T}}\right]\right\}^{1 / 2}
$$

Remembering that Smagorinsky used $L=$ the grid spacing $\Delta$, we see that we recover the familiar Smagorinsky model with the model constant

$$
C_{\mathrm{s}}^{2}=\left(\frac{A_{\mathrm{t}}^{3}}{D_{\mathrm{t}}}\right)^{1 / 2}\left(\frac{L}{\Delta}\right)^{2}
$$

Since $C_{\mathrm{s}} \approx 0.2$ for $L=\Delta$, and this value is known to within a factor of two or so, equation 61 provides a loose constraint on compatible values of $A_{\mathrm{t}}$ and $D_{\mathrm{t}}$ in the transport model.

C. E. Leith (private communication, c. 1991) suggested using a similar argument to extend the Smagorinsky model to include buoyancy creation and a compressive eddy work term. The simplified transport equation 58 picks up the buoyancy creation term $\mu_{t} \mathcal{B}$ on the right hand side. This $\mathcal{B}$ should include rectification or any other modifications to the basic formula. Then including the buoyancy creation term in the eddy viscosity means replacing $S^{2}$ in equation 58 with $S^{2}+\mathcal{B}$. Then we find

$$
\mu_{\mathrm{t}}=C_{\mathrm{s}}^{2} \tilde{\rho} L^{2}\left\{\left[S^{2}+\mathcal{B}+\left(\eta_{d} \nabla \cdot \overline{\mathbf{u}}\right)^{2}\right]^{1 / 2}-\eta_{d} \nabla \cdot \overline{\mathbf{u}}\right\} .
$$

with $\eta_{d}=0$. The terms proportional to $\eta_{d}$ are additional empirical terms introduced by Leith to allow the Smagorinsky viscosity also to play the role of an artificial viscosity for shock waves. Leith suggests values of $C_{\mathrm{B}} \approx 1.43, C_{\mathrm{S}} \approx 0.2$, and $\eta_{d} \approx 10$. The parameter $\eta_{d}$ should be set to zero for low Mach number flows. These parameter values should be considered tentative. 


\section{Conclusions}

The present model addresses only a few of many issues that must be resolved in developing a truly predictive model of compressible turbulent flows. In the present study, we used simple kinematic models to justify adding pressure gradient terms to the turbulent heat flux and to the buoyancy creation of turbulence kinetic energy. These terms are not obvious from superficial examination of the governing equations. These modifications have several implications.

- The pressure gradient term in the turbulent heat flux arises from $P d V$ work. Since no analog of that term appears in the species continuity equations, no analog appears in the species turbulent diffusion fluxes at this level of approximation. Any countergradient term in those fluxes must arise somewhere else, and the present study does not rule these out.

- The buoyancy creation term must also have the adiabatic term, as must the often-used $\nabla P \cdot \nabla \rho$ criterion for stability. Failure to include this term may result in nonphysical flow predictions as a result of violating the second law of thermodynamics.

- When buoyancy effects are present, the original Smagorinsky model must be modified to include them, as suggested in section 7 .

- As shown by Deardorff, there is reason to believe that there are additional terms missing from the present closure models, and further research is needed.

\section{Acknowledgments}

I thank John Ramshaw for many useful discussions and James Park for his comments on the manuscript. This work was performed under the auspices of the U. S. Department of Energy by University of California Lawrence Livermore National Laboratory under contract No. W-7405-Eng-48.

\section{References}

[1] P. H. Gaskell and K. H. C. Lau, "Pressure-containing correlations in variable density and turbulent reacting flows," Comb. \& Flame 73, 215 (1988).

[2] J. W. Deardorff, "On the direction and divergence of the small-scale turbulent heat flux," J. Meteorology 18, 540 (1961). 
[3] J. W. Deardorff, "The counter-gradient heat flux in the lower atmosphere and in the laboratory," J. Atmos. Sci. 23, 503 (1966).

[4] J. W. Deardorff, "Theoretical expression for the countergradient vertical heat flux," J. Geophys. Res. 77, 5900 (1972).

[5] V. P. Starr, "Physics of Negative Viscosity Phenomena," (McGraw-Hill, New York, 1968).

[6] L. H. Aller and S. Chapman, "Diffusion in the sun," Ap. J. 132, 461 (1960).

[7] R. B. Bird, W. E. Stewart, and E. N. Lightfoot, Transport Phenomena (Wiley, New York, 1960).

[8] S. Chapman and T. G. Cowling, The Mathematical Theory of Non-Uniform Gases (Cambridge University Press, London, 1953).

[9] J. D. Ramshaw, "Self-consistent effective binary diffusion in multicomponent gas mixtures," J. Non-Equilib. Thermodyn. 15, 295 (1990).

[10] J. D. Ramshaw and C. H. Chang, "Ambipolar Diffusion in Multicomponent Plasmas," Plasma Chem. Plasma Proc. 11, 395 (1991).

[11] T. Montmerle and G. Michaud, "Diffusion in stars: Ionization and abundance effects," Ap. J. Suppl. 31, 489 (1976).

[12] L. D. Cloutman, "Compressible Turbulence Transport Equations for Generalized Second Order Closure," Lawrence Livermore National Laboratory report UCRL-ID-134075, 1999.

[13] D. R. Stull and H. Prophet, "JANAF Thermochemical Tables, 2nd ed.," U. S. Department of Commerce/National Bureau of Standards report NSRDS-NBS 37, 1971.

[14] M. W. Chase, J. L. Curnutt, A. T. Hu, H. Prophet, A. N. Syverud, and L. C. Walker, "JANAF Thermochemical Table, 1974 Supplement," J. Phys. Chem. Ref. Data 3, 311 (1974).

[15] M. W. Chase, Jr., C. A. Davies, J. R. Downey, Jr., D. J. Frurip, M. A. McDonald, and A. N. Syverud, "JANAF Thermochemical Tables, Third Edition, Parts I and II," Supplement No. 1, J. Phys. Chem. Ref. Data 14 (1985).

[16] S. Kaniel and A. Kovetz, "Schwarzschild's criterion for instability," Phys. Fluids 10, 1186 (1967). 
[17] J. P. Cox and R. T. Giuli, 1968, Principles of Stellar Structure, Vol. 1. Physical Principles (New York: Gordon and Breach).

[18] D. D. Clayton, Principles of Stellar Evolution and Nucleosynthesis (McGraw-Hill, New York, 1968).

[19] S. Chandrasekhar, An Introduction to the Study of Stellar Structure (University of Chicago Press, Chicago, 1939).

[20] P. Ledoux, "Stellar models with convection and with discontinuity of the mean molecular weight," Ap. J. 105, 305 (1947).

[21] U. Schumann, "Subgrid scale model for finite difference simulations of turbulent flows in plane channels and annuli," J. Comput. Phys. 18, 376 (1975).

[22] F. J. Rogers, F. J. Swenson, and C. A. Iglesias, "OPAL equation-of-state tables for astrophysical applications," Ap. J. 456, 902 (1996).

[23] F. W. Sears, An Introduction to Thermodynamics, the Kinetic Theory of Gases, and Statistical Mechanics, Second Edition (Addison-Wesley, Reading, MA, 1953).

[24] W. C. Rivard, T. D. Butler, and O. A. Farmer, 1975, in Proceedings of the Fourth International Conference on Numerical Methods in Fluid Dynamics, ed. R. D. Richtmyer (Berlin: Springer-Verlag), p. 334.

[25] Cloutman, L. D., "A New Estimate of the Mixing Length and Convective Overshooting in Massive Stars," Ap. J. 313, 699 (1987).

[26] L. D. Cloutman, The LUVD11 Large Eddy Simulation Model, Lawrence Livermore National Laboratory report UCRL-ID-107128, 1991.

[27] L. D. Cloutman, "Numerical simulations of the heat transfer and decay of turbulent swirling flows," Computers \& Fluids 17, 437 (1989).

[28] J. Smagorinsky, "General circulation experiments with the primitive equations: I. The basic experiment," Mon. Weather Rev. 91, 99 (1963).

[29] J. W. Deardorff, "On the magnitude of the subgrid scale eddy coefficient," J. Comput. Phys. 7, 120 (1971). 